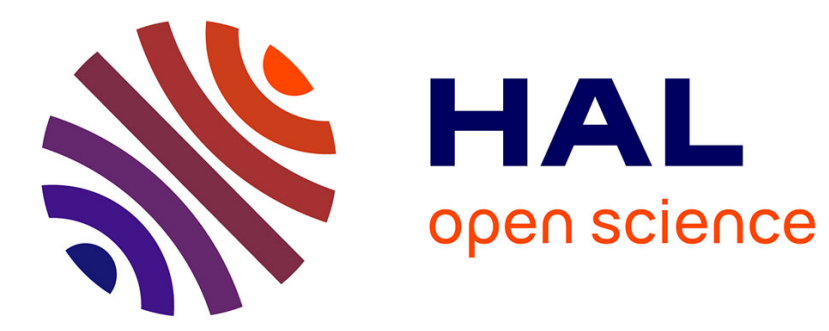

\title{
Role of sketching in conceptual design of car styling
}

Carole Bouchard, Améziane Aoussat, Robert Duchamp

\section{To cite this version:}

Carole Bouchard, Améziane Aoussat, Robert Duchamp. Role of sketching in conceptual design of car styling. Journal of Design Research, 2006, 5 (1), pp.116-148. 10.1504/JDR.2006.010810 . hal00915137

\section{HAL Id: hal-00915137 \\ https://hal.science/hal-00915137}

Submitted on 6 Dec 2013

HAL is a multi-disciplinary open access archive for the deposit and dissemination of scientific research documents, whether they are published or not. The documents may come from teaching and research institutions in France or abroad, or from public or private research centers.
L'archive ouverte pluridisciplinaire HAL, est destinée au dépôt et à la diffusion de documents scientifiques de niveau recherche, publiés ou non, émanant des établissements d'enseignement et de recherche français ou étrangers, des laboratoires publics ou privés. 


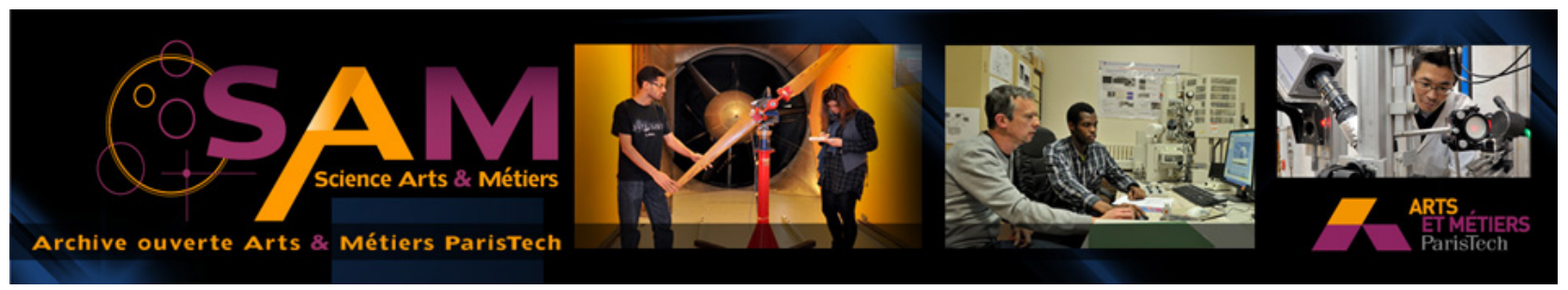

Science Arts \& Métiers (SAM)

is an open access repository that collects the work of Arts et Métiers ParisTech researchers and makes it freely available over the web where possible.

This is an author-deposited version published in: http://sam.ensam.eu Handle ID: .http://hdl.handle.net/10985/7604

\section{To cite this version :}

Carole BOUCHARD, Améziane AOUSSAT, Robert DUCHAMP - Role of sketching in conceptual design of car styling - Journal of Design Research - Vol. 5, n¹, p.116-148 - 2006 


\title{
Role of sketching in conceptual design of car styling
}

\section{Carole Bouchard*, Améziane Aoussat and Robert Duchamp}

Laboratory of New Products Design, Ecole Nationale Supérieure des Arts et Métiers, 151 Bd de l'Hôpital, 7013 Paris, France

E-mail: bouchard@paris.ensam.fr

E-mail: ameziane.aoussat@paris.ensam.fr

E-mail: roduchamp@wanadoo.fr

${ }^{*}$ Corresponding author

\begin{abstract}
This paper aims at studying the role of sketching in car styling. Prior studies have shown that sketches play a major role in the early phases of design. A definition of the intermediate representations and mental representations will enable a better understanding of how sketches are produced. Two distinct experiments are proposed: a series of interviews of 40 professional car designers and a protocol study with six junior car designers. A comparative study demonstrated that two main phases appear in the specific car design process, including 'routine' and 'non routine' activities linked with external inspiration sources.
\end{abstract}

Keywords: sketching activity; conceptual design; mental and graphic representations; car styling.

Reference to this paper should be made as follows: Bouchard, C., Aoussat, A. and Duchamp, R. (2006) 'Role of sketching in conceptual design of car styling', J. Design Research, Vol. 5, No. 1, pp.116-148.

Biographical notes: Carole Bouchard is an Assistant Professor at the Ecole Nationale Supérieure des Arts et Métiers in Paris France. Her research activities are centred on design trends watch methods, engineering emotional design, Kansei engineering, creativity and innovation. She develops methodology in design science, mostly centred on the study of the cognitive activity of the designers in the early design process.

Améziane Aoussat is the Head Manager of the Laboratoire Conception de Produits et Innovation at the Ecole Nationale Supérieure des Arts et Métiers in Paris. His role is in the management of the overall research activities and industrial collaborations. He is the scientist responsible for the laboratory and supervises the pedagogy of research thesis and the training of engineers.

Robert Duchamp is the Head Manager of the Laboratoire Conception de Produits et Innovation in France. His role is in the management of the network 'Presence and Innovation', including several sites and laboratories. These laboratories are all involved in research in innovation: the laboratory product design and innovation in paris, the pole méditerranéen de l'innovation in marseille, and the laboratory presence and innovation in laval. 


\section{Role of sketching in conceptual design of car styling}

\section{Introduction}

During the past ten years, a change from traditional to digital tools has taken place in engineering design: traditional models tend to be replaced by digital mock-ups. CAD tools proved to be useful in the phases of embodiment and detail design. They enabled a quicker development with increased performances. In the traditional design process, more or less structured forms of pictorial information are produced by the designers. CAD corresponds to the traditional production of high structured forms of pictorial representations such as plans or sections. Concerning the sketching activity, we can find in the traditional process more unstructured forms related to creativity and innovation in design. Today, the designers prefer to use traditional sketches for the earliest phases of conceptual design, because of their ability to fulfil their natural and intuitive needs during their activity. The effort-saving character of sketches allows the designers to concentrate more on the creative side of conceptual design. New digital tools related to sketching activity may be able to keep these properties.

The integration of new digital tools in the early stages of development is the most crucial because attributes such as the shape and the spatial configuration are depicted to the product during this phase. It is assumed that in the design process the earlier phases like task clarification or embodiment design will have the greatest impact on the development costs of the future product.

In the following paper the major hypothesis is that a study of the cognitive activity of designers could help to suggest recommendations for the elaboration of adapted digital design tools, aiming to support the earlier phases of the design process, task clarification and conceptual design. Therefore an investigation is needed with an emphasis on the role and specific effect of traditional tools as sketches.

In this paper, two distinct experiments are presented: a survey based on a series of interviews of 40 professional designers and a protocol study with six junior designers (students) in the specific context of the motor design process. Car design according to us seems to be a privileged application field because it works according to a very complex and formalised process in terms of intermediate and external representations.

\section{Theoretical background: conceptual design and its representations}

During design activity, the aim of designers is to change their environment. By observation and interpretation of the results, the decision is taken if changes are needed to be made on this environment. Their concepts may change according to what they are seeing in consequence of what they have done (Gero, 2002). Design activity involves a process where designers produce solutions in both individual and collective ways. The generation of new solutions is based on the interpretation and assimilation of various intermediate representations. Until recently little attention has been paid to the function that such representations have during conceptual design, besides Purcell and Gero study (Purcell and Gero, 1998). A few years ago they proposed a review paper which constitutes an essential starting point. The aim of the paper was to review several areas like working memory, imagery reinterpretation and mental synthesis, to examine their implications for design research generally given a particular emphasis on sketching design. Many relevant point of views were emphasised in this paper of major researchers in the field such as Gross et al., Goldschmidt, Goel, Schön and Wiggins, Suwa and 


\section{Bouchard, A. Aoussat and R. Duchamp}

Tversky, Finke, Katz and others (Purcell and Gero, 1998). The discussion revealed that drawings, are associated with reinterpretation or the emergence of new ways of seeing and drawing, where reinterpretation cues access to other relevant knowledge and allows inferences to be made. In the case of drawings, the knowledge accumulation can be perceptual and associated with the physical attributes of the design represented in a drawing. Another issue was linked to the working memory. The role of drawing in design can be grasped in considering the design process as a recursive sequence of activities involving thinking, imagery, drawing and re-interpretation, accessing different types of knowledge in long-term memory, where reinterpretation concerns images of perceptually ambiguous figures and mental synthesis of forms. Finally, authors argued that solving design problem should exhibit decomposition, involving different types of reasoning, sequential processing at a macro scale and the use of external memory aids.

Another study of Römer et al. was aimed to show the dominance of sketches as external aid and the increasing use of CAD in addition to the production of models. The designers confirmed that external representations do not only serve as external information storage but as a important external aids for example solution development, testing and communication. The study particularly underlined the transverse and specific functions of the external representations used in design. The function of communication was identified as a transverse and main function for all types of external representations. Sketches were linked with the specific function of solution development and support of memory, models with solution development, CAD with solution testing and complex models with the checking of requirements.

In order to grasp the reasons of the designer's attraction for the traditional tools during sketching, the first thing to do was to investigate the specific representations of the conceptual design phase. One major concern in the notion of representations is their nature. In fact intermediate representations can be internal or external. The designer has to transform his internal mental representation into an external one which can be shared, interpreted and used by other people. We describe the intermediate representations as a mental or physical one used during the design process from the beginning to the final product.

\subsection{Internal representations in conceptual design}

Mental representations can be defined as incidental constructions created in a given situation in order to meet the requirements of the current task (Richard, 1983). They differ from perception in that they either constitute an evocation of objects that are absent at the time, or they double the perception of present objects by referring to other images (Piaget, 1948). The mental representations used during the design activity are contextual and linked to specific goals. In that way they are temporary and evolutionary: they will change as soon as the situation will evolve. They are quickly available and provide an operational memory, like internal models elaborated by the designer in order to treat situations (Falzon, 1969). In these representations, the symbols are maintained internally within the device (Norman, 1991). This internal model is the result of a design based on the analysis of situated data and on the evocation of memorised knowledge, according to the notion of 'situatedness' quoted by Gero (2002). The concept of operative image introduced by Ochanine (2001) specifies the notion of representation by simultaneously introducing understanding or evocation as well as operability: image of an object that adequately reflects its major distinguishing features and which is 


\section{Role of sketching in conceptual design of car styling}

by the same token appropriate for human intervention. Related to design activity, the initial representation which stems from the brief's treatment will gradually evolve as the uncertainty is reduced, thanks to the application of the acquisitive process of interpretation (construction of a representation which is compatible with expectations), and finally as representations building by monitored knowledge (activation and transfer of knowledge from long term memory towards working memory). A design problem is characterised by the simultaneous construction of the problem and the solution, with a multitude of solutions and incomplete data. The designer gradually constructs an individual representation of this problem/solution space. In that way, Schön and Wiggins (1992) defines design as a conversation between the material and the designer where appreciation is noticed in the materials, the goals of the design process is changed as a result of appreciation during design. The result will be in any event original, particular and different from those that would have been produced by a panel of various experts in the same field, even if they had the same constraints (Bisseret et al., 1988; Carrol and Moran, 1991).

\subsection{Early external representations in conceptual design: sketches}

Graphic realisation makes it possible to fix a mental image and make it physical, in a space that gradually gets closer to the produced space. In the context of a teamwork involving different skills, the physical object expresses an unambiguous representation of which the reading is immediate by all the team, whatever their origin or function. Besides its operative character, realisation improves communication. Moreover, the graphic displays are useful because they allow to show permanent representations. Sketches are usable in an intuitive and cost-efficient way without unnecessary loss of time (Römer et al., 2001). They allow odd definitions due to the incompleteness of product definition within the early stages (Römer et al., 2001). They effect a memory and enable communication. Finally, they are preferred because of their quickness of execution, fuzzy definition, impact of communication and freedom to concentrate on creative tasks. Schön and Wiggins (1992) defines the move as the basic unit of the design process, being a transformation to the average design, and the move experiment as making a move and seeing the results. Sketching activities can be related to moves in terms of whether there is active sketching within a move, whether the designer is thinking about a sketch and copying off a sketch. Which means that the designer is either using the support of a sketch or using is own mind creativity (Purcell and Gero, 1998). Sketches are usually the first formal support used by the designers. In the specific field of car design, the graphic can be seen as a system of signs that results from the relation between a signifier (the sketch) and a signified in reference to (the vehicle). The process of graphic materialisation corresponds to the refining of this system of signs in time. Sketching embody abstract and high-level design ideas, allowing a degree of uncertainty about particular physical attributes to exist. Vehicle attributes such as shape and spatial configuration will be depicted during sketching.

The major hypothesis is that a study of the cognitive activity of designers could help to suggest recommendations for the elaboration of adapted digital design tools, aiming to support the earlier phases of the design process, task clarification and conceptual design. Therefore an investigation is needed with an emphasis on the role and specific effect of traditional tools as sketches. 


\section{Bouchard, A. Aoussat and R. Duchamp}

In this paper, two distinct experiments are presented: a survey based on a series of interviews of 40 professional designers and a protocol study with six junior designers (students) in the specific context of the motor design process. Car design according to us seems to be a privileged application field because it works according to a very complex and formalised process in terms of intermediate and external representations.

\section{Questions}

We were interested in the following questions in order to analyse the role of sketching in conceptual design of car styling:

- what are the input data integrated in the conceptual design process by the car designers and the conscious mental images they perceive during sketching?

- what is the succession of tasks in the whole car design process and the resulting external representations?

- what is the succession of tasks and the resulting external representations during the specific task of sketching?

\section{Experiments}

We studied the earliest phase of the design process from the briefs refining to the realisation of the first sketches by means of analysis, which were undertaken with car designers. The study included two complementary separate phases:

- the questionnaire

- the protocol study.

\subsection{Study 1: questionnaires}

The questionnaires were focused on our goal to identify the input data integrated in the design process by the car designers and the conscious mental images they perceive during sketching.

\subsubsection{Subjects (see Table 1)}

The questionnaires were distributed to 150 designers. We only obtained 40 answers 12 from professional designers of three European car motor companies, and 28 from students in car design (see procedure P7). So therefore we gathered a first set of information through the survey, the sample was composed mainly of students $(75 \%)$ and professional designers $(25 \%)$. Professional designers ranged from artistic to technical backgrounds. The very few replies of professional designers, compared to that of students made it impossible to do a rigorous comparison between these two different groups, even if we propose qualitative comments linked to this point, in the part 3.1.4, related to the three different expertise levels. This study was more focused on the information that designers use and produce than on the understanding of design expertise. However 


\section{Role of sketching in conceptual design of car styling}

some differences will be emphasised in the results part. Three variables were considered in the analysis: the expertise degree in car design (three levels were distinguished), the background of the designers (distinction between three schools), and the mother tongue (influence on the vocabulary employed and the significances attached to the various words or concepts). They were considered as expert's professionals currently working in the branch, intermediate experts the students having acquired a concrete skill in car styling by the participation in several industrial projects, and the novice students were those not yet having acquired concrete experience in the field. Considering the students, 14 were novices and 14 were intermediate experts (ending their degree course). Other factors, as the sex or the age of the subjects were not taken into account.

Table 1 Designers profiles

\begin{tabular}{lccc}
\hline & Novices & Intermediate expertise & Seniors \\
\hline Art center (Europe) & 3 & 4 & 2 \\
Coventry (England) & 7 & 5 & 4 \\
Ensaama (France) & 4 & 5 & 6 \\
\hline Total & 14 & 14 & 12 \\
\hline
\end{tabular}

From this survey we can single out six additional characteristic points revealing specificities of the profession:

- $\quad$ there were no females

- designers were relatively young (20-35 years)

- $\quad$ we can find very cosmopolitan nationalities in each car design school (Italian, Japanese, French, Spanish, German, Greeks, Slovenians ...)

- the initial background, before the choice of the design, was various (artistic, applied in industrial design or technical with engineers and technicians)

- the training in car design reveals diversities in terms of learnt disciplines, methods, techniques and tools. There are autodidacts in small proportions (two in our survey)

- there are manual designers (rather from an artistic formation) or designers using digital tools (rather from technical training).

The population of car designers is singular by its diversity, in term of various initial training, nationality, orientation towards manual or digital tools and its conformity in term of age and sex.

A synthesis of the contents of the specific trainings in three establishments offering a specialisation in car design (Art Center Europe, Strate College and Coventry University) reveals a common list of eight sciences and techniques related to the knowledge and skills considered as essential by the car designers:

- $\quad$ engineering aerodynamics, manufacturing techniques and materials

- history of car design and industrial design

- $\quad$ product and car design

- 2-3 D materialisation techniques (sketching, manual and digital modelling) 


\section{Bouchard, A. Aoussat and R. Duchamp}

- social and human sciences (ergonomics, marketing)

- communication

- design strategy and design methodology.

Training related to manual and digital tools differs noticeably according to the schools: $30 \%$ of the subjects said they learnt the use of digital tools in certain schools, while $70 \%$ admitted they only used manual $2 \mathrm{D}$ and $3 \mathrm{D}$ modelling techniques). The values and lessons transmitted by the school culture act upon the styling activity by a particular treatment of the formal characteristics, giving specific orientations on particular targeted fields teaching different methods. They are visible by the practical application of rendering or modelling rules as colours mixing for instance. Besides the theoretical teaching, a significant part of the studies is devoted to the development of individual skills and the realisation of school collective projects, but also industrial projects allowing to keep up a privileged link with the car industry.

\subsubsection{Instruments}

The same questionnaires were distributed to the designers, whatever their current occupation and background. The only difference was the language used, giving birth to three different versions in English, French and German. The questionnaire was composed of eight pages with a short explanation of the goals of the study at the beginning (see Figure 1). The following contents were a set of 60 questions, with a percentage of half and half between closed and open ones.

The expected contents were linked to the following information:

- identity of the designer: age, sex, nationality, professional experience, design fields of the company, background, initial training with name of the schools

- culture: style and design definition by the designer, and finally the word he is used to employ, vocabulary used to define the car parts, most familiar words used with colleagues

- manner of sketching (sequence with the different views, parts of vehicle, volumes, mental images and integrated data)

- inspiration sources (fields, evolution)

- brand image (semantic adjectives describing the brand image, specifications in terms of lines, colours, volumes, values of the company, own values of the designer, importance of items as actuality, evaluation, firms or schools culture during sketching, technical and consumer related items)

- manner of integrating the technical constraints, influence of the technical constraints on styling and vice versa

- manner of integrating the consumer related constraints as socio styles

- consideration of the expressivity (type of view, most expressive parts of the vehicle, most expressive volumes, biomorphism, relative importance of function, aesthetics, symbolism for the designer, familiar tools manual or digital, preferred tools)

- impact of the criteria of the sketching evaluation process on sketching

- examples of success or failure in car design according to the designer. 


\section{Role of sketching in conceptual design of car styling}

Figure 1 Questionnaire addressed to the designers

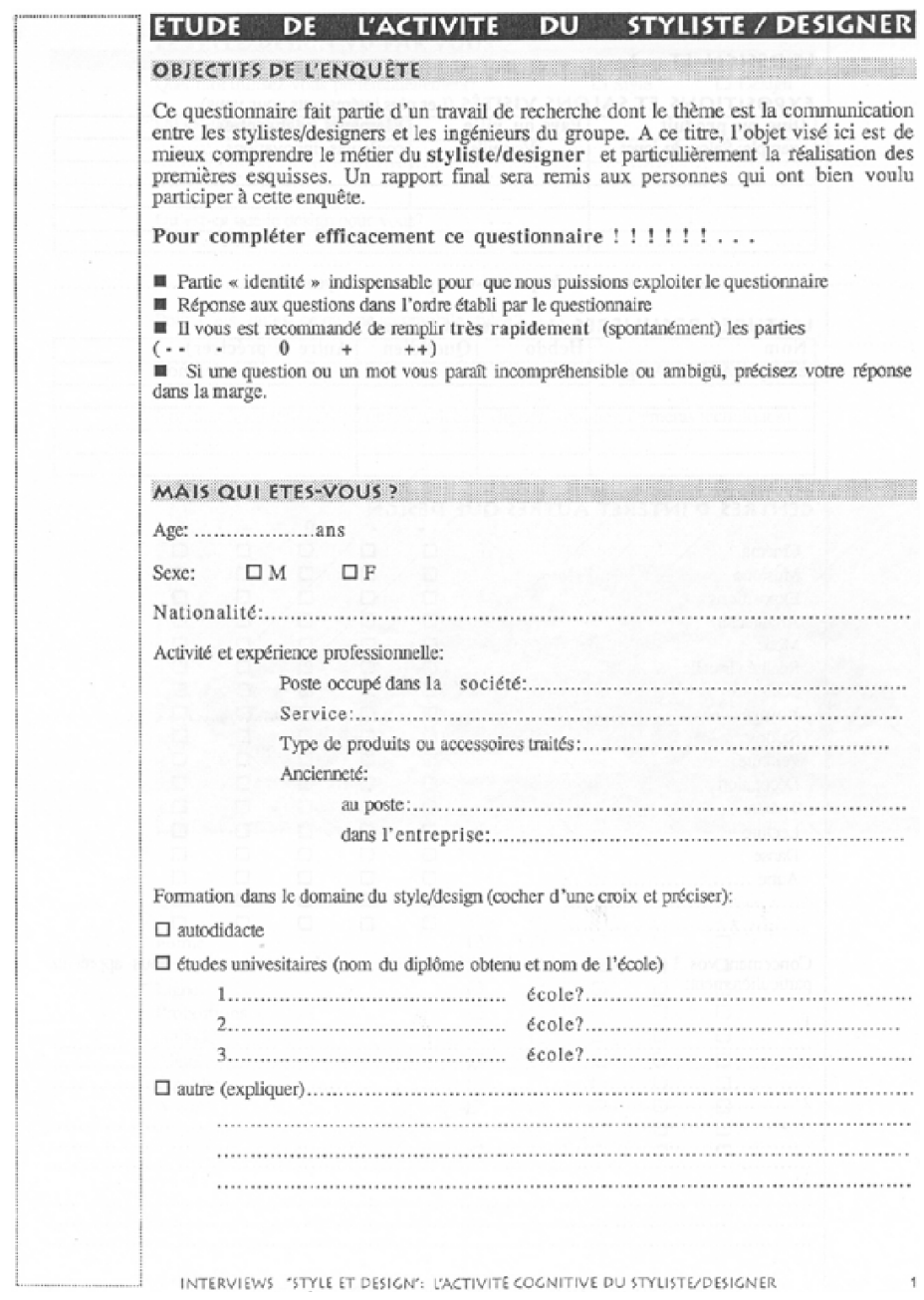

\subsubsection{Procedure}

The questionnaire was built on the base of interviews with two designers. It was finally validated by three pilot tests with one designer for each country in England, Germany and France. The questionnaires were directly given to the designers by a coordinator in each design department and school. Questionnaires were under paper form and the coordinator had to make a follow-up to the designers before collecting the answers.

The results of the questionnaires were categorised by a content analysis including the number of occurrences for each category. We recorded information related to the own design process of each designer including a focus on his conscious mental images and inspiration sources. The detailed examination of the answers of the questionnaire proceeds of a contents analysis by the way of basic statistical indices $(\%$, average, row ...). 


\subsubsection{Results}

The analysis and synthesis of the results provided the following information:

- minimal knowledge of the actors (motivations, training, expertise ...)

- representation of the field of expertise

- global description of the design process (selection criteria, implied actors, media used)

- categorisation of integrated information as project constraints that can refer to consumers characteristics (age, social level, sex, expertise, lifestyles ...) or object characteristics (semantics, sensoriality, usefulness), various influences; however it was not possible with the questionnaire to identify precisely the moment of integration of these data in the design process.

Design was emphasised by the interviewed designers as

- the integration (compromised, connection) of varied fields and knowledge

- the method referring to the various research processes and procedures and data processing.

One massive and exclusive 'yes' was expressed by the various designers concerning the use of the term design (76\%), rather than that of style, only used by English experts designers. In the different schools related to our sample, we can thus speak about 'design', a field located between styling and engineering.

Three distinctions can be made concerning the types of treated projects: interior/exterior conveys, design global/detailed, and conveys serial/concept car.

After consideration of the data contained in the brief, an information search process is systematically performed by the designers to fill in the problem space with non dimensioned values. Strong visual elements are extracted from various sources of inspiration, references and influences, linked with their personality and intuition. The data integrated in the conceptualisation come from different fields, more or less directly linked with the initial domain. When asked about their conscious sources of inspiration, $62 \%$ of the interviewed designers reported several sources. The following areas were spontaneously mentioned in Table 2 :

Table 2 Percentage of spontaneously quoted sources of inspiration of the designers

\begin{tabular}{lclrlr}
\hline Universe of arts & $(\%)$ & Universe of nature & (\%) & Universe of products & (\%) \\
\hline Music & 10 & Animals & 20 & Automotive & 95 \\
Journeys & 5 & Felines & 3 & Aeronautics & 20 \\
Cinema (Science-fiction) & 5 & Insects & 2 & Architecture & 10 \\
Painting & 4 & Vegetables & 1 & Furniture & 5 \\
Drawing & 4 & Plants & 1 & Fashion design & 5 \\
Graphic arts & 4 & Flowers & 1 & Telecommunications & 4 \\
Sculpture & 4 & Minerals & 1 & Hi/fi & 3 \\
Cartoons & 2 & Stone & 1 & Video & 2 \\
& & & & Food & 1 \\
\hline
\end{tabular}




\section{Role of sketching in conceptual design of car styling}

These sources of inspiration vary in compliance with the type of project or vehicle to be worked on and the personality of the designer. Particular items are sought out across these domains such as

- impressions and feelings connected with global perception (creativity, modernity, innovation, sensations, spirit, trends)

- $\quad$ stylistic elements (aesthetics, proportion, structure, shape, line, volume, relief, colour, motifs, textures)

- technical characteristics (aerodynamics, engineering performance, technologies, materials, internal details)

- consumer-related items (adhesion values, semiotics, ergonomics, sensory characteristics, taste).

Information details derive from a deliberate information search effort carried out in order to meet the specifications of the brief and from the more or less conscious re-activation of knowledge previously memorised during various activities possibly going beyond the professional context. The result of the information research is translated preferably by the visual shape (trend panels, various sketches, etc.), the textual mode being used to highlight the key points. The designers carry out a more or less systematic and organised investigation, from a simple glance through to the making up of trend panels and databases connected with the initial domain or other domains of influence. This is performed in part by visits to showrooms and exhibitions and by image integration. The investigation process carried out by subjects in areas other than car design remains highly subjective and personal. These areas include various domains of activities, fields of interest or leisure activities to which the designer refers to on an irregular basis, for which frequency and performance are relatively variable. Showrooms or exhibitions visited and regular readings involve transport, with a major representation of this initial domain, architecture and many other fields that are not necessary shared (see Table 3 ).

Table 3 Percentage of visited show rooms, exhibitions and of regular readings of the car designers

\begin{tabular}{llc}
\hline Visits of show-rooms and exhibitions & Transport & $72 \%$ \\
& Architecture & $10 \%$ \\
& Plastics & $8 \%$ \\
& Objects & $6 \%$ \\
& Painting & $4 \%$ \\
& Information technologies & $4 \%$ \\
& Furniture & $1 \%$ \\
Regular readings & Transport & $58 \%$ \\
& Architecture & $10 \%$ \\
& Consumer information & $8 \%$ \\
& Design & $5 \%$ \\
& Fashion & $3 \%$ \\
& Popular science & $3 \%$ \\
& Graphic design & $3 \%$ \\
\hline
\end{tabular}


Common mentioned fields in terms of visited show-rooms, exhibitions and regular readings are

- transports (mainly cars with $92 \%$, bikes, utilitarian vehicles, heavy trucks, water vehicles, aeronautics)

- the field of architecture with $10 \%$ of regular readings and exhibitions; concerning exhibitions, one finds beside plastics $8 \%$, objects $6 \%$, painting $4 \%$, data processing $4 \%$ and furniture $1 \%$, while regarding regular readings, design $8 \%$, fashion or popular science $3 \%$ and graphic design $2 \%$.

The choice of these references changes with the time for $60 \%$ of designers, depending on the type of project or object to design, the designer himself according his own values, interests, moods (30\%), or even the abundance and renewal of the source $(21 \%)$, finally fashion, influences or more particularly what is perceived as appreciated by the press and the consumers (16\%). Among the designers, $15 \%$ thought that there is not any evolution of the inspiration sources (whatever the expertise level was) and $25 \%$ did not answer to this question. The designer resorts frequently to several sources of inspiration. As such, here are some examples of sources used in the framework of specific students projects:

- bear cub in design of baby seats

- jewellery, furniture, old coaches of 18 th century in a specific design of a ceremonial coach for the queen

- African buses, African values and lifestyles, nature, natural houses, animals, in a design project for a three wheels vehicle for South Africa

- generally, for the design of a new model for a company, various old models, for proportions and aesthetic rules ... with an avant-garde treatment

- food

- philosophy, history.

Added to this according to the designers, the treatment of lines and volumes is itself influenced by the personal expression (signature sometimes very important), by the reference to car styling as a discipline (reference to famous stylists), by technical parameters and lifestyles (quite important), and evaluation criteria of sketches as well as corporate or schools values (less important).

With respect to the integration of the sources of inspiration in conceptual design, questions referred to the preliminary images, which are used by the designers. The designers mentally visualise various conscious images before to sketch the first lines. As Figure 2 shows, these may be classified in four categories.

The first category is related with images from the sources of inspirations $(62 \%)$ integrated for their shape, silhouette and appearance. They are usually linked to recent objects of design $(30 \%)$ or fields such as transport design with vehicles or other transport facilities recent or old, competitors, prototypes (22\%), human or animal $(20 \%)$, and other fields as nature, fashion, sports, and architecture. They will be used essentially through an analogical reasoning process allowing visual translation or transformation like morphing. 
Role of sketching in conceptual design of car styling

Figure 2 Verbalisation of the designers concerning their mental images

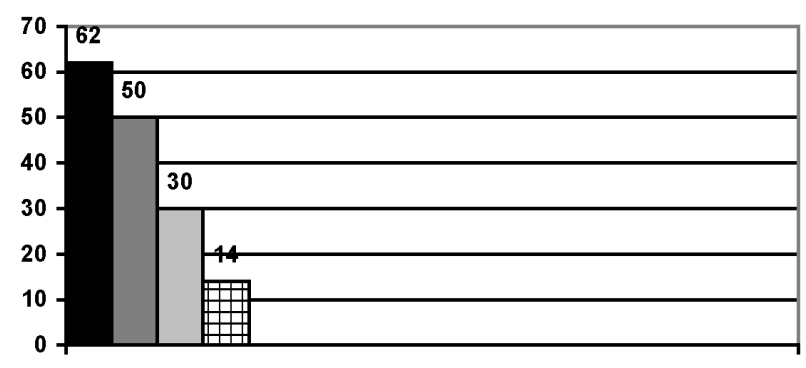

The second category concerns ephemeral and global images of the whole vehicle to be drawn with general characteristics (50\%). This image is very evolutionary, dynamic and highly related to the notion of proportions and balance. It will provide the emotional impact.

The third category includes ephemeral and images of parts of the vehicle and constituents as wheels, vehicle lines or internal mechanics (30\%).

The fourth category seems to be directly linked to mental images reflecting the conceptualisation work of the shape of the current thought emerging concept as the intersection of simple volumes, or graphic ideas and geometrical features (14\%).

These categories of mental images are used simultaneously. They are all ephemeral and cannot be considered as mutually exclusive. According to a few designers $(10 \%)$, it is possible to sketch the first lines without having any conscious mental image and so try to infer one by drawing lines. In this case the mental image results from an unpredictable graphic image. The findings of the questionnaire allowed the deduction that the sources of inspiration play an important role during the conceptualisation, and more precisely for the elaboration of the global shape. The designers are able to identify consciously most of them.

After showing that various sources of inspiration are present when generating the global shape and main parts of the concept vehicle, connected with the global perception of the concept vehicle, as well as stylistic elements, technical characteristics and consumer-related items, the next questions of interest were concerned with the succession of tasks in the whole car design process and the resulting external representations, with a particular focus on the specific task of sketching.

\subsection{Study 2: protocol study}

\subsubsection{Subjects}

The protocol study aimed to study the early phase of sketching. It implicated only students because of the difficulty to observe professional car designers in a very secret environment. Regarding this point, we met certain constraints during the realisation of the study. The most restrictive was the banning of the use of any observation method of the design activity in builders styling departments: professional stylist have not been observed during their activity.

The protocol involved six graduate male students of the car design department of the Coventry University. For professional car designers, the design process can differ because of the use of specific professional tools (true to scale sketches) or of the process itself more constraining in companies. But the general approach follows approximately 


\section{Bouchard, A. Aoussat and R. Duchamp}

the same steps. Lecturers act both as partners and customers of the future designers in the design process, like engineers or senior design managers with designers in the car industry. They are mainly here for evaluation. At the end of their project in collaboration with companies, students are able to realise the professional process while integrating their own identifiable style. Further comparison between students and professional designers was not made in the framework of the experiments.

\subsubsection{Instruments}

In order to perform the protocol study, the following instruments were defined and used:

- a semi-directive interview

- a videotaping (see Figure 3).

The semi-directive interviews consisted of a mixture of structured questions and answers style meeting. We formulated the questions based on our goal to gather information about:

- the succession of tasks in the traditional design process

- the resulting external representations during sketching

- the semantic correlation between words (given in a simulated brief) and the corresponding design elements produced by the designers. More precisely, the expected contents of the semi-directive interviews mentioned in the question grid were the following:

- description of the design process (different stages, different representations, evaluation process, media used)

- description of the sequence of information integration (nature of the information, moment of integration)

- semantic projected dimensions by the designers according to the brief.

The discussion had to be supported by several concrete examples.

Figure 3 Protocol instrumentation
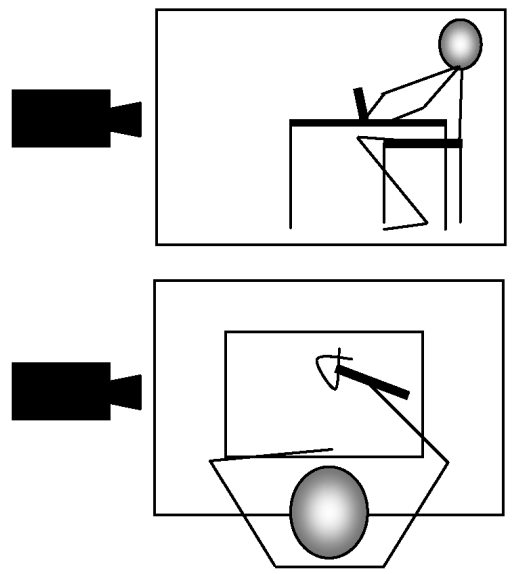


\section{Role of sketching in conceptual design of car styling}

Concerning the observation, two simultaneous video recordings were done, one for a global view allowing to take in account the position with the table, the head and the trunk of the designer, and the other in a restricted field for scrutinising the attitude, the hands movements and the graphic production. In order to perform the sifting through the data, the whole body movements have been observed thanks to a slow playing of the videotapes. A gathering and analysis of the generated tracks by the designers in their sketches has been added to this observation. It allowed to facilitate the video analysis, and to obtain complementary data as dimensions. The subjects worked with the usual traditional tools they use at the beginning of a design project during the protocol study: black pen and $\mathrm{A} 3$ paper block.

\subsubsection{Procedure}

The protocol study aimed to study the early phase of sketching. The protocol study was divided into two sections consisting of a semi-directive individual interview during about 45 minutes and the observation of a design session during one hour with video and sound recordings. We organised individual work sessions with the six students allowing to realise both the interviews and to go deeper into the results by the video and sound recording. The designers worked individually without any correspondence between them and their colleagues during the protocol study. The six participants were voluntary and had acquired the same amount of knowledge and experience in car design. They were at the end of their degree course and were considered as intermediate experts. The interviews were done on the base of an evaluation grid of interview, recorded with a tape recorder and fully transcribed. The analysis was qualitative. The video observation aimed to record the simulated sketching activity during 40 minutes, and during the 20 other minutes, to extract the semantics projected by the designer.

The design assignment to the students was the following: firstly during the first 40 minutes, "to draw a family model, in 15 minutes". It was very open in order to let students concentrate more on the copy of their process during a real project, without strong constraints, which could require a long time; secondly during the 20 other minutes, "to produce quick sketches in order to illustrate words". The given words were brands and specific values.

The results of the interviews and observations were completed by the examination of sketching books related to industrial projects and showing an organised series of sketches coming from specific briefs of European car companies. This enabled us to extract some data concerning the early design process as the information integrated depending on the type of project and its various phases, as well as the objects signified attributions. Routines were identified during the graphic realisation.

\subsubsection{Results}

The analysis and synthesis of the results provided the following information: results provided the following information

- description of the succession of tasks during the early design process with design phases, succession of drawn objects, building mode

- resulting external representations during sketching with their evolution

- correspondence between the semantics projected by the designers and design elements. 


\section{Bouchard, A. Aoussat and R. Duchamp}

The interpretation of these results allowed us to define more deeply the correspondence between the information integrated in the process by the designer with the initial assignment and the semantics linked to the representations.

Beside, important elements have been formalised related to the movements of the designer during the graphic production, taking in account subtasks as simulation, generation or modification of graphic tracks. Information regarding the block handling (vertical, horizontal and rotations) revealed itself very useful in order to characterise the activity. In fact this item can have an impact on the recommendations for the design of new digital tools. For example the current graphic tablets do not allow this kind of movements. So they require a specific training. Other information related to injury times gave information about the auto-evaluation process, which can be detected thanks to the association of variables as 'a straight posture', 'to stand up straight', and the vehicle design strategies. Results allowed to formalise certain angles of the semantic dimension of the concept, with the examination of the graphic tracks like vehicle parts, considered also according to their signifiers.

\subsubsection{Succession of tasks in conceptual design process}

The design process followed by the participants is described below. The following description of the opportunist approach of the designers should be understood as iterative process even though it is written here as a sequence.

\section{A: Reception of the brief}

The initial brief can be very open before to become progressively more and more closer: for example, to find a collective transport media for the city.

B: Collation of information about the brief during a couple of days (inspirational influences and existing cars or concept cars)

Designers often take their inspiration in science fiction, natural world (animals ...), aeronautics, architecture, and of course transport and automotive design to get a visual mental representation of the future possible. For matching their inspiration to the brief, they start up by a brainstorming to think about all issues involved.

C: Research of initial ideas with sketches during a week (whatever format):

First sketches are not close to the brief. They only show a silhouette (lines and a little pastel) with the wheels, profile of the vehicle, glass areas, sometimes body sections and joint lines, allowing the designer to fix one of him dynamic visual thoughts. The process is repeated till the designer is satisfied with some ideas.

\section{D: Evaluation of research sketches for selection of the best ideas}

The first evaluation is the auto evaluation of the designer. After that comes a collective evaluation from the other designers. Designers select firstly the sketches themselves, with colleagues opinion considered. Shape with proportions and functional response are important criterions for the initial selection.

\section{E: Formalisation of final ideas during many weeks (A3 or A2 formats, see Figure 4)}

The elaborated sketches are more detailed and give more feeling, even if engineers cannot use the information at this stage. The first view drawn is often the front $3 / 4$ view. 


\section{Role of sketching in conceptual design of car styling}

With this kind of view, it is relatively easy to draw because it is almost a $2 \mathrm{D}$ representation: it is easier to draw lines, which follow the natural flow of the hand as volumes. It gives information about front, side and rear. So it is a good compromise between the ease of use and the many expressed information. Wheels are the first drawn elements because they help to give the right proportions. They constitute a reference with the ground level. To summarise, the designer begin with a rich $2 \mathrm{D}$ representation and imagine then the vehicle turning around its central point, producing fix images by sketching means. Drawing allows to freeze the dynamic thought process on a particular representation. The detailing is important too, but comes after the global shape.

Figure 4 Final sketches (designer mat noone)

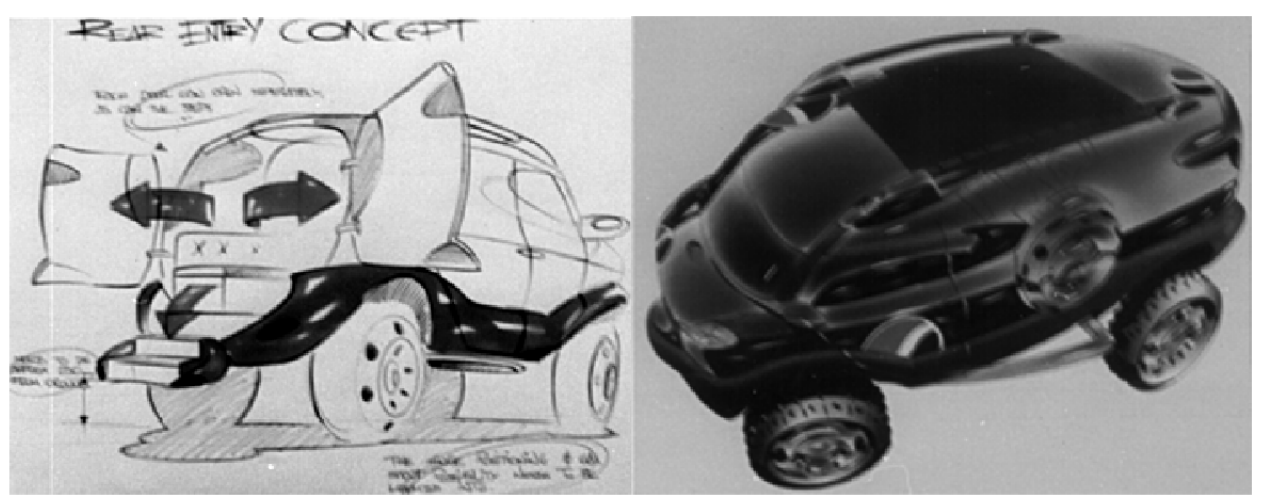

\section{F: Final sketches evaluation: reviewing brief and finding areas of interest}

After the research of the best ideas comes the whole integration of the brief. Final ideas are discussed with lecturers and the brief is refined while students try to convince them.

\section{G: Development of final concept}

After the selection of the theme, engineering drawings and the render drawn package are then used to integrate visually and measurably the main constraints as dimensional packaging (engine location, people) and engineering simultaneously (technical data, physical questions, problems of scale, product size in relation to real life, objects, realistic believable shaping and time).

\section{H: Generation of $1 / 5$ scale tape drawing}

This type of drawings allow the designer to reproduce the lines of the chosen sketches with black adhesive tape from $3 \mathrm{~mm}$ to $6 \mathrm{~mm}$ in side elevation, for eventual modifications in real time.

\section{I: Production of a small Polystyrene model (one day)}

\section{J: Production of a 1/5 scale model in clay (many weeks, see Figure 5)}

Clay model give a 3D representation easy to modify. To better show the style lines, tape adhesive is used on the 3D model too. When modifications happen at this stage, they are done simultaneously on the 2D tape drawing and on the 3D model. Students often need quick modifications, and it can happen till the last stages. The main modifications are style details. 


\section{Bouchard, A. Aoussat and R. Duchamp}

Figure 5 Model (designer mat noone)

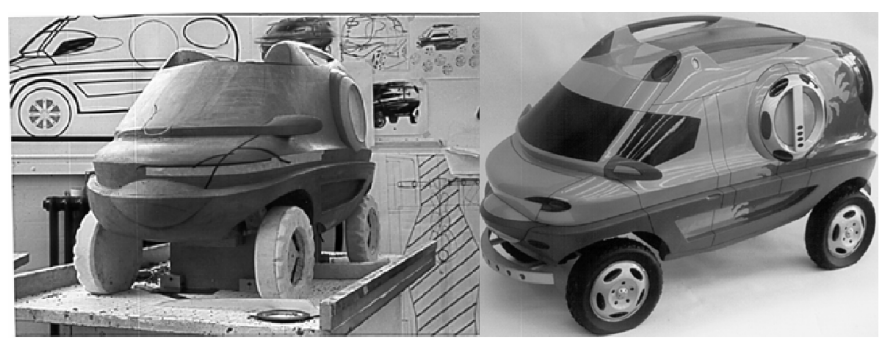

\subsubsection{Resulting external representations during sketching}

The results of the experimental protocol concern the creative process involved during the production of the first sketches. Sketches are initial visual graphic 2D representations aimed to communicate a 3D mental image. They may only be self intended and concerned with major features rather than details. They content the minimal necessary draw lines to communicate the message (corporate, model, character, personal expression ...) without any measured information. Eyes fill in the missing details and the mind create the whole image. Designers usually start the sketch phase with a $3 / 4$ front view: this commonly used type of sight enables the best compromise between the quantity of signified information and the convenience in which the signifier can thus be created.

As shown in Figure 6, lines can be exaggerated thanks to this technique so as to generate a major emotional impact without however distorting basic proportions. Here, one finds information about the front, the profile, the top. Moreover, it helps in clarifying style partially defined at this stage with specific lines. This type of sight enhances the plan of horizontal lines (or close to the horizontal), and follows the natural movement of the hand. A perspective sight is then reconstituted from the $3 / 4$ view in order to have a better representation of the volume (see Figure 7).

Figure 6 First sketches in the automotive design process

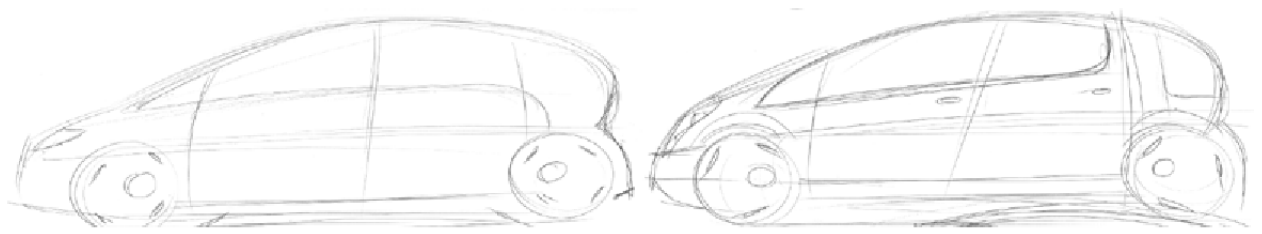

Figure 7 Perspective sight

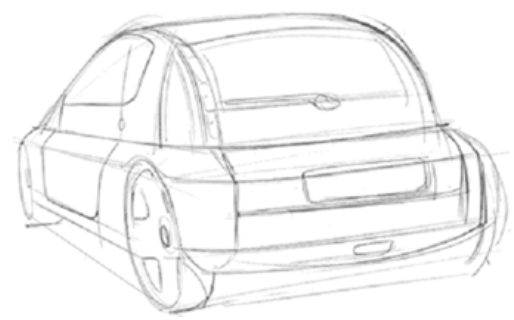




\section{Role of sketching in conceptual design of car styling}

This is generally done from a point of view that offers more impact and is related to dynamic tension with an angle of perspective. The latter is determined in relation to the type of vehicle. Sensation is strengthened when perspective is exaggerated, however, this kind of perspective generates a loss of dimensional information.

The views, which are the least revealing about identity and bear the least meaning are orthogonal sights (see Figure 8), which nonetheless supply a direct measure.

Figure 8 Orthogonal sight

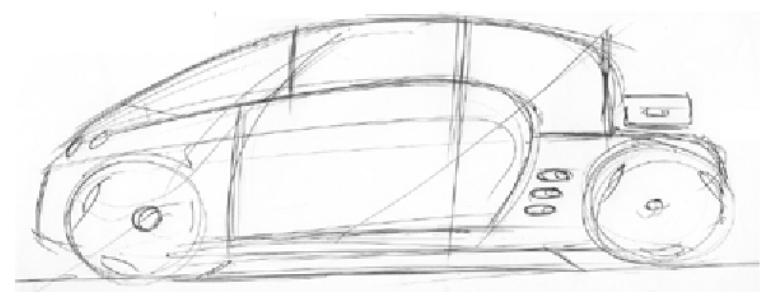

In terms of representation strategy, one distinguishes a 2D strategy (see Figure 9) adopted by five subjects, according to which the same $3 / 4$ front view is reproduced several times, traced or remembered. Lines are then refined during the representation and the details are added. Concerning the 3D strategy, we could not extract any routine because of the poor common inputs we had with the only one other subject. However, interesting information were found out: $3 / 4$ view is declined according to other point of views on the same sheet or on the different sheets, as if the vehicle was revolving on itself. Various views are simultaneously worked on, in correspondence or in a sequential way. Whatever the chosen strategy is, the first represented forms are geometrically easy to memorise (horizontal lines, circles, ellipses). They can be more complex however they will be memorised through experience and repetition.

Wheels are the first objects represented in a routine way (by six subjects) on a horizontal line for the ground or bottom package. The representation of all the wheels fixes certain attributes of the vehicle as horizontal proportions, structure, and type. The package bottom line can be slightly oblique with regards to the ground: this denotes the vehicle's attitude. The global object is horizontal, and the component 'wheels' are oriented in such a way as to suggest movement by the vehicle's direction. The dimensions and orientation of the wheels create criteria of impressiveness. One or several horizontal lines as the top line of the bumper, the package bottom line, as well as the middle package line, represent the lower part of the vehicle. The position of these lines is connected to the type of vehicle. Until this stage in the representation process, we obtain forms that are geometrically simple and easy to remember.

The creative part entirely starts on this base. The plan of the global or good shape starts with the definition of the vehicles outline. Fixing the global proportions of the vehicle is thus made possible. The first drawn curve generally circumscribes the vehicle's front part profile (grill, bonnet, bumper, and the vehicle's false front), with little details such as the grill and logo. At this stage, the windscreen's base and the glazed bays can be drawn. Then, a second curve delineates the profile of the front windscreen and the roof. This curve is then extended to the back of the vehicle so as to define the windscreen and the body rear. The outlines of the front and rear windscreen are in all events limited to a right segment or a bow of a concave curve. At this stage, good shape is fixed. One can recognise the type of vehicle, sometimes its nationality, and even its brand. The plan of 


\section{Bouchard, A. Aoussat and R. Duchamp}

the outline is followed by a readjustment of the lines, which completes the general architecture in that the vehicle's uprights and other sections are pencilled in. Details and components are then added. This precedes another readjustment of the lines for optics, number plates, air intakes, grill, logo, assembly zones ... Treating details corresponds to a more thorough inspection that provides the means to complete the bases shape.

Figure 9 Early sketching process: the succession of tasks according to the 2D strategy

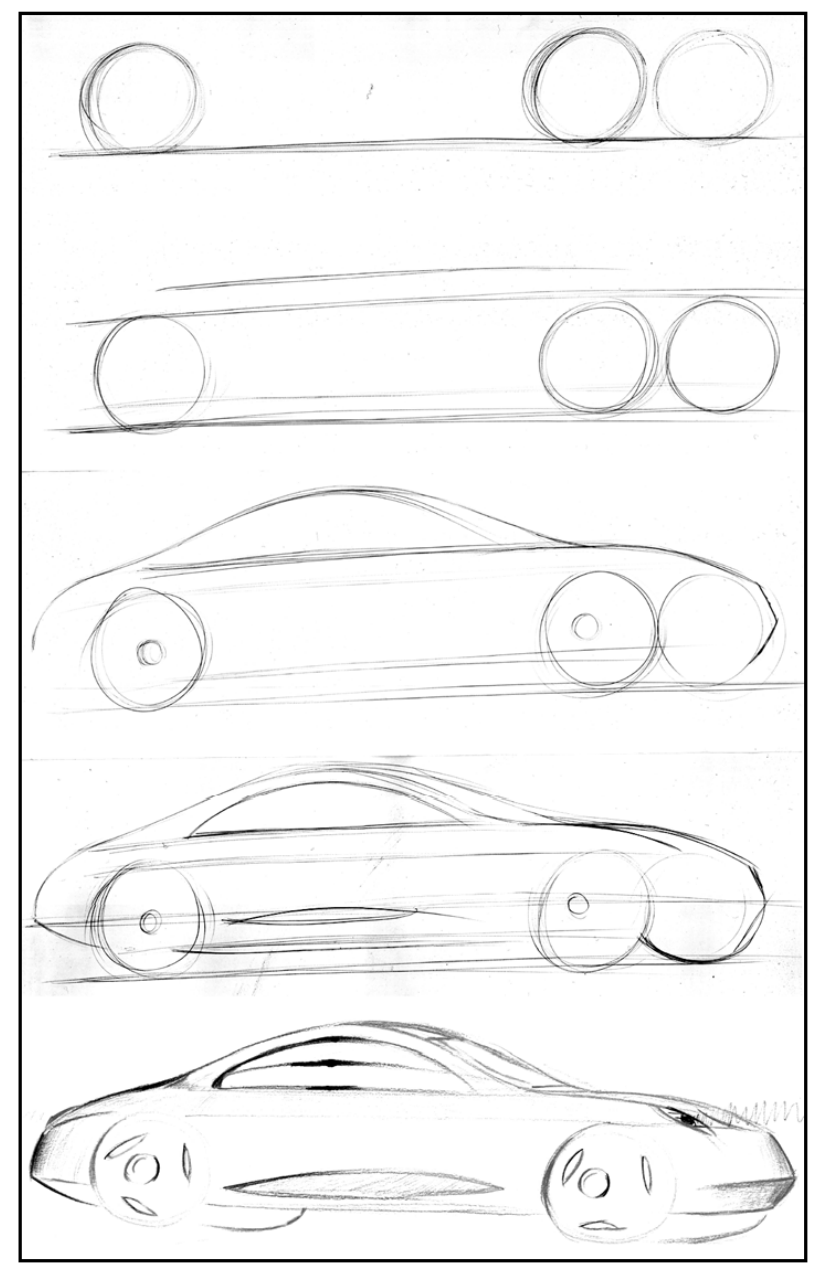

\subsubsection{Correspondence between given words and styling tracks produced by the designers}

Design activity can be seen as the progressive production of signs trough the successive intermediate representations. After a first formalisation of the early design process through the different tasks and representations, we sought after the signs projected in the tracks the designers produced from a given brief. We examined these signs according to two complementary ways: 
- the interpretation of the sketched vehicle helped by the verbal description of the six student designers

- the interpretation of the quick sketches they drawn spontaneously from given words characterising the brand image and the values that could be mentioned in the brief.

A qualitative analysis allowed us to establish the correspondence between words expressing values and corresponding sketches produced by the designers (here are some examples).

According to the results (see Figure 10), the first value open-mindedness seems to be correlated to a friendly aspect, denoted by a smiling attitude of the vehicle face or very avant-garde lines. The value of power is shown through the denoted aggressiveness of the vehicle or the perceived power of the motor with sportive models. The value of dynamism is shown through a very reduced slant or a front in arrow form. Finally the value of safety refers to balance presented by a frontal and profile symmetry. Consideration of the whole results lead us to the conclusion that outside the brand, values or semantic adjectives are mainly signified through the type of model (sportive, comfortable), the general proportions (balance, slant), and the front face expression (smiling, aggressive).

Figure 10 Correspondence between values words and tracks produced by the designers

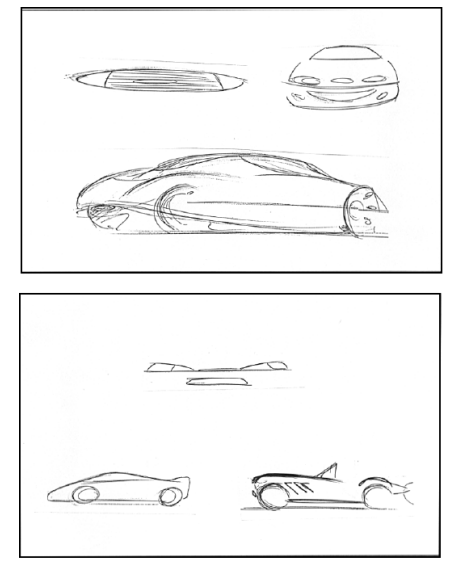

Open-mindedness

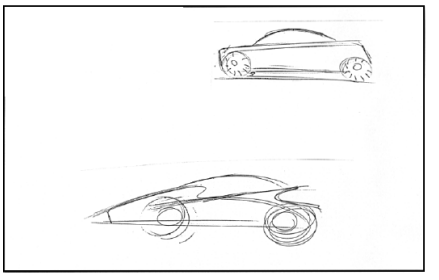

Dynamism

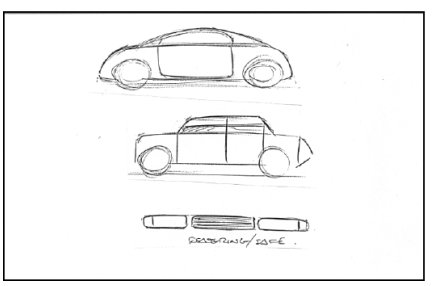




\section{Bouchard, A. Aoussat and R. Duchamp}

Another link between values and semantics comes from the brand image (see Figure 11). It can be very strong in the case of BMW or Peugeot brands, or lighter when it is only shown through the logo for instance.

Figure 11 Formal characterisation of BMW brand image by the six subjects

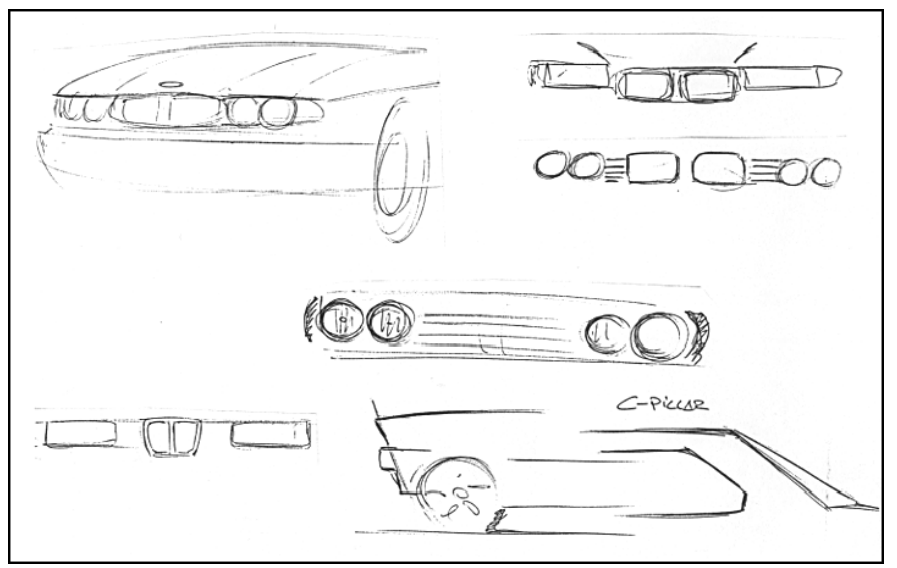

Considering the brand BMW, the form of the grid (often named nostrils by the designers) and that of perfect round headlights appear as the most representative elements of the brand revealing a strong identity. Then the designers emphasised the importance of the bonnet form, bumpers, rear-view mirror and of the general styling treatment with form and lines, volumes and semantic adjectives like sporty, German, strong, aggressive, serious, traditional and robust.

Beside, the strongest signature of the brand Peugeot (see Figure 12) comes from the grill and the headlights as well, and after that the bonnet and the bumper. The lines appear as very important enlighteners of the brand too, with in particular the profile (side, elevation, silhouette, profile, bold-crease-along-side), the logo, the front, the three box design, the tensed lines and forms, styling and semantic words like elegance, dynamism, modernity, sobriety and classical.

Figure 12 Formal characterisation of Peugeot brand image by the six subjects

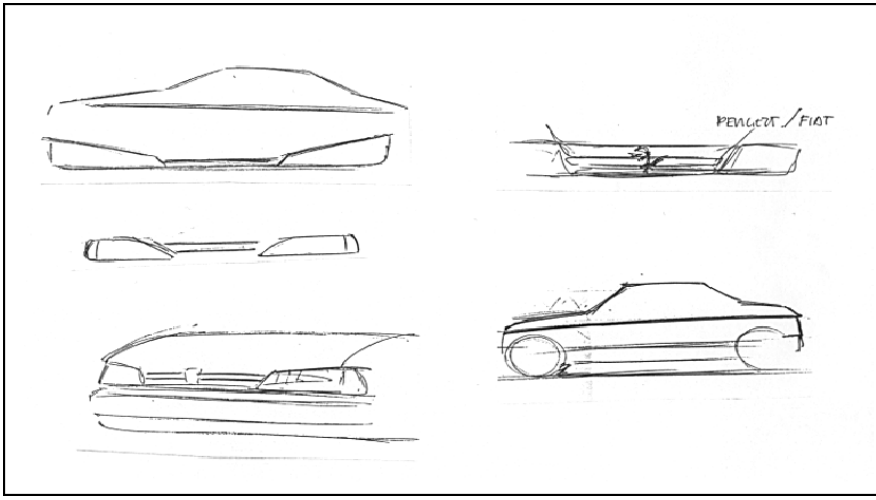

Source: Bouchard (1997) 


\section{Role of sketching in conceptual design of car styling}

We can observe a great coherence between these results and general additional results obtained in the questionnaire with the whole sample of designers. The corresponding question was the following: "to give words in order to indicate the most important elements signifying the brand image". In fact we can claim that the most important parts of the vehicle showing the brand image are the grid, the badge and the headlights. In fact, as shown before, these elements were spontaneously drawn when we asked to the six students to sketch the most important elements denoting the brand image (for BMW or Peugeot for instance, see Figure 13).

Figure 13 Formal characterisation of BMW brand image by the six subjects

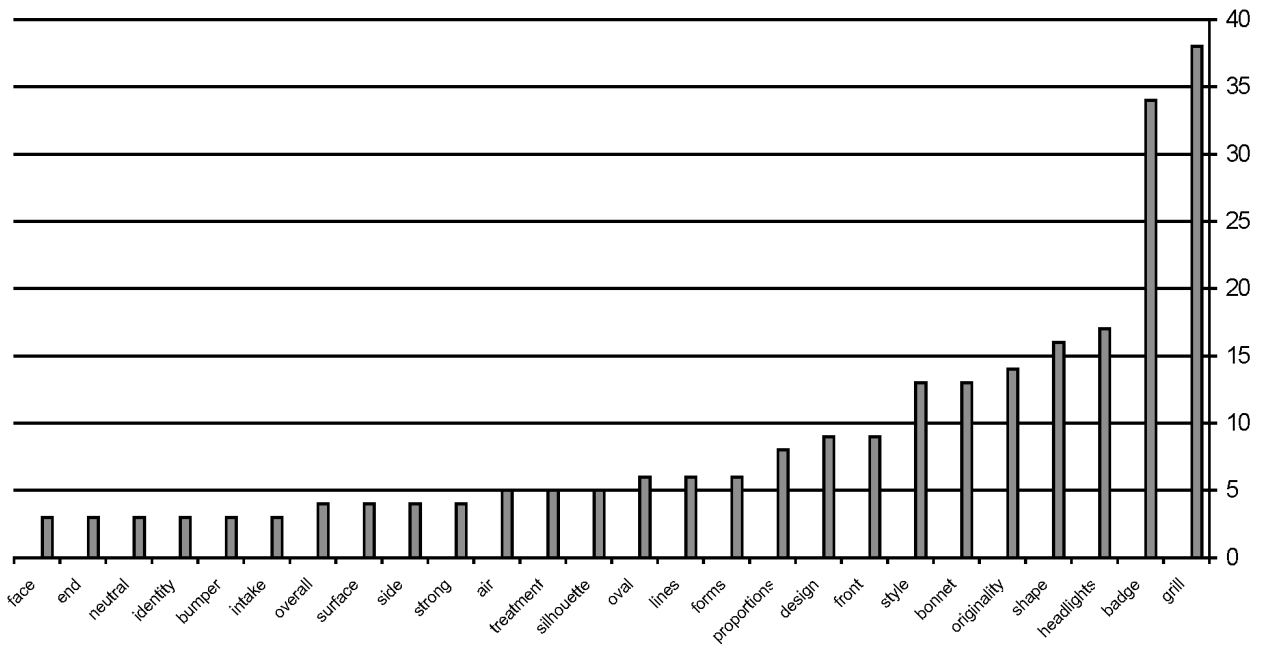

In conclusion (see Figure 14), brand image can be expressed by a specific treatment of vehicles components or of vehicles parts, more shown on the front or the profile of the sketched vehicle (front, profile, rear has not been represented). The rear of the vehicle seems to be less crucial in the early sketching process (it corresponds to the last view drawn) and it has not been mentioned as an important recognition element. The treatment of formal characteristics like proportions, lines, or form expressed during styling have a big impact as well. Both are linked to the semantic expression, which will favour a particular brand recognition too (strong/VW). We therefore conclude that the important items allowing recognition of the briefs specifications linked to the brand image and projected values are the following:

- definition of the vehicle

- $\quad$ styling treatment

- $\quad$ semantics.

These items are very inter dependant and refer to the notion of significant and signified. It is through them that we analysed the sequence of representations presented in 3333 in order to define a generic model of the signs sequence in early design (p24). 


\section{Bouchard, A. Aoussat and R. Duchamp}

Figure 14 Signs system expressed in sketches

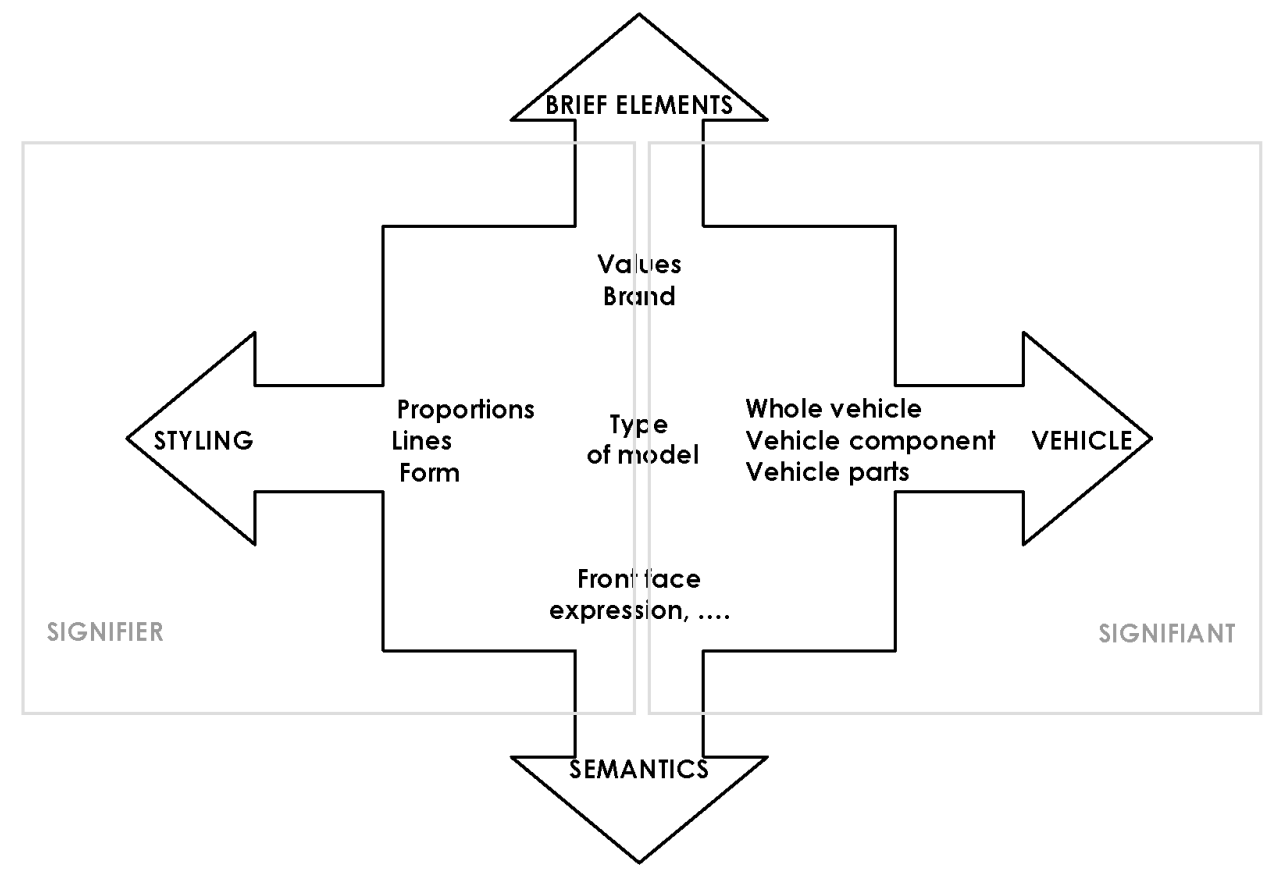

\section{Discussion}

In order to better understand the role of drawing in conceptual design, we identified the succession of tasks and the resulting external representations in the global process. This approach allowed us to consider the conceptual design process as a sequential processing at a macro scale aided by the use of external memory aids (Purcell and Gero, 1998).

In fact, the design process can be seen as the diachronic transformation of a space problem into a space solution (see Figure 15), according to a recursive cycle in which a partial reconfiguration takes place from the space problem to intermediate solution spaces at every synchronic representation level (brief + inspiration sources, sketches, CAD, simple models, complex models) (Bouchard and Aoussat, 2002). This partial transformation first occurs by way of the mental representations of the designers. Then the actions are performed through a feedback process involving both a generation and evaluation phases through the different external representations. External representations act as mediators between us and the world, both in execution between actions and the resulting changes to the world state, and in perception between changes in the world and our detection and interpretation of the state (Norman, 1991). They can be taken as virtual objects which operations are performed to be reflected onto the real future product. 
Role of sketching in conceptual design of car styling

Figure 15 Description of a materialisation cycle

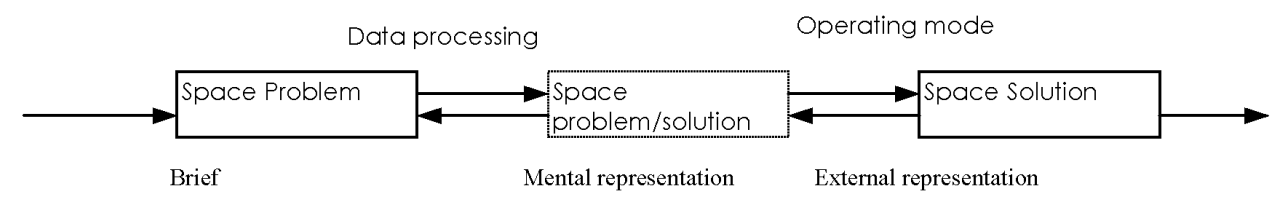

At any time in the process, designers must be able to assimilate and treat a large volume of information without losing sight of their goals. This equivalence characterises the problem/solution space. The quantity of information will be progressively transferred into the space solution, through the mental and physical representations, as follows (see Figure 16):

Figure 16 Representations in the design process

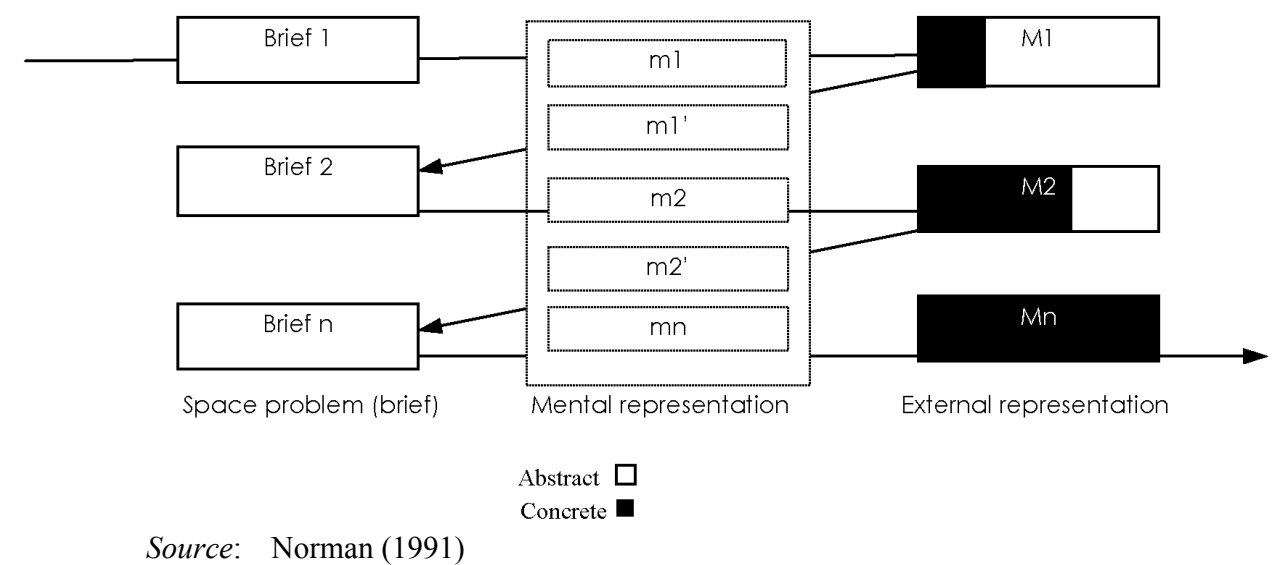

The design process consists of reducing abstraction through the use of various successive levels of representation integrating more and more constraints. It is characterised by Wang as a conceptualisation including an iterative cycle of mental solutions shown with a given problem (Wang, 1995), where

- the problem is ill defined

- the selection of a solution or partial solutions enables the reduction of uncertainty, keeping in mind the necessary level of vagueness in modifications during subsequent phases (Lebahar, 1992, 1993)

- new constraints are then added by preserving shape and initial ideas

- the display of a new physical representation is going to generate new ideas and new solutions.

Abstract finalised information is transformed step by step into material information, by way of intermediate mental and physical representations. MC Kay proposes a generalisation of the theory of information: information is what forms or what transforms a representation (Birtley, 1995). One can make a comparison between the transformation of images and the representations generated by a message containing information. The information modifies the representations, so one can measure communication 


\section{Bouchard, A. Aoussat and R. Duchamp}

by measuring these changes. By the way of externalisation, the constructive memory (Gero, 2002) becomes a physical system which supplies an external memory, and which modifies perception in a decisive way. In this sense, the space problem as well as the space solution are punctually made explicit by the production of signs (see Figure 17). Each cycle Brief1, m1, M1 corresponds to a state of representation. The evolution of the Brief is defined by the transformation of ends in purposes, from purposes to objectives, from objectives to criteria, criteria in more and more concrete technical specifications. Mental representation corresponds partially, as does the physical model. By the definition of every model in a set of attributes A11, A12, .., process $\mathrm{P}$ is then characterised by the succession of cycles: Brief1M1 (A11, A12, A1n), Brief 2M2 (A21, A22 ... A2n), Briefn Mn (An1, An2, Ann) ... ). Process develops a representational system of information (objectives) in a signs system (solutions), of which recognition and evaluation allow the production of new signs. Signs emanate from the process of the mental composition of an imaginary object into an object readily comprehensible by anyone. The space solution has in particular the passage of iconic signs in plastic signs. It is established by a succession of modelled forms.

Figure 17 Design process considered as a sign sequence

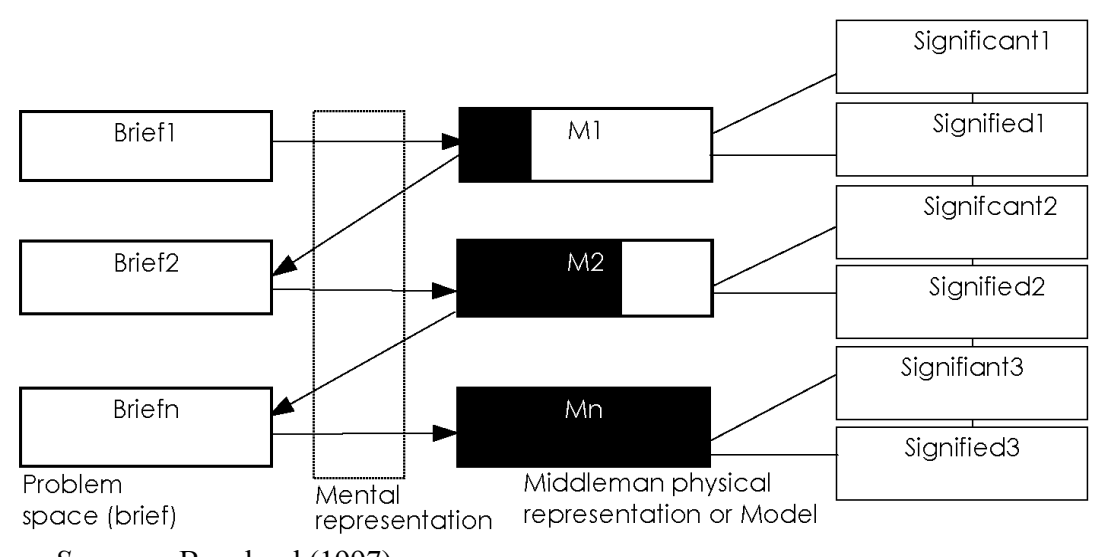

Source: Bouchard (1997)

From the results of Section 4.4.2, each modelled form M1, M2, representing the object is bound by a known referent (see Table 4), that is a particular and unnecessary real object member of a class which has its own appropriate physical characteristics, where the significant is linked to a referent by a relation of transformation (isomorphism, specific to the cultural character of the recognition codes). This representational system supports the conceptual design activity where the signified codes will be gradually integrated to the sketches.

Whatever the strategy is (2D or 3D), the representation of the wheels and of the ground follows a routine noticed by all the subjects. At this stage, major attributes can be fixed as proportions, structure, and the type of vehicle. We can imagine that routine steps can integrate more elements than the wheels in the case where the corporate graphic symbols are very strong. We made the experiment with students but professional designers may memorise more characteristics related to the brand image and generate more constrained sketches in that sense (see Figure 18). 
Role of sketching in conceptual design of car styling

Table 4 Alterations of representations as systems of signs during the creation of the first sketches (2D strategy)

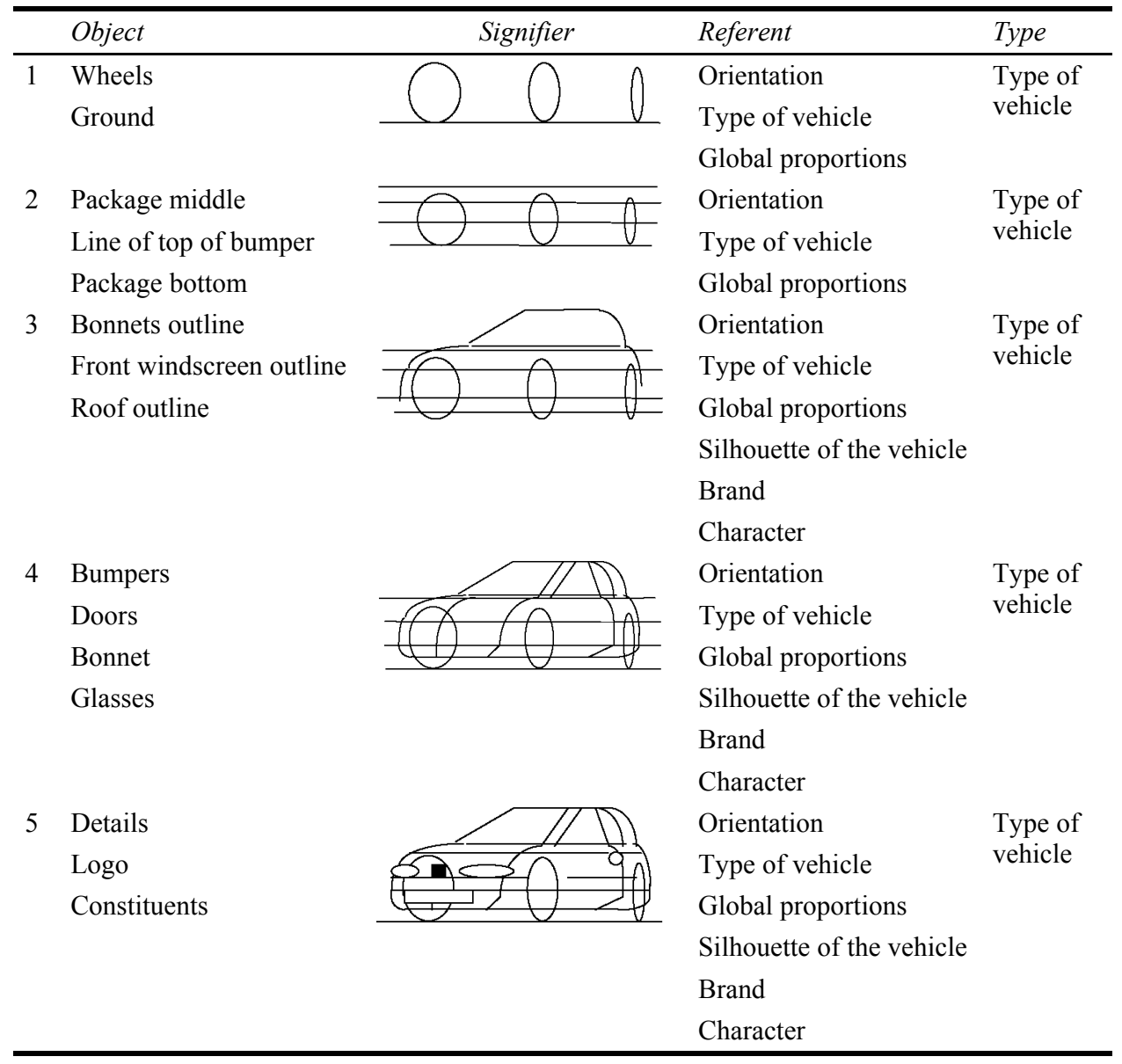

Source: Bouchard (1997)

Figure 18 Formal characterisation of BMW brand image by the six subjects

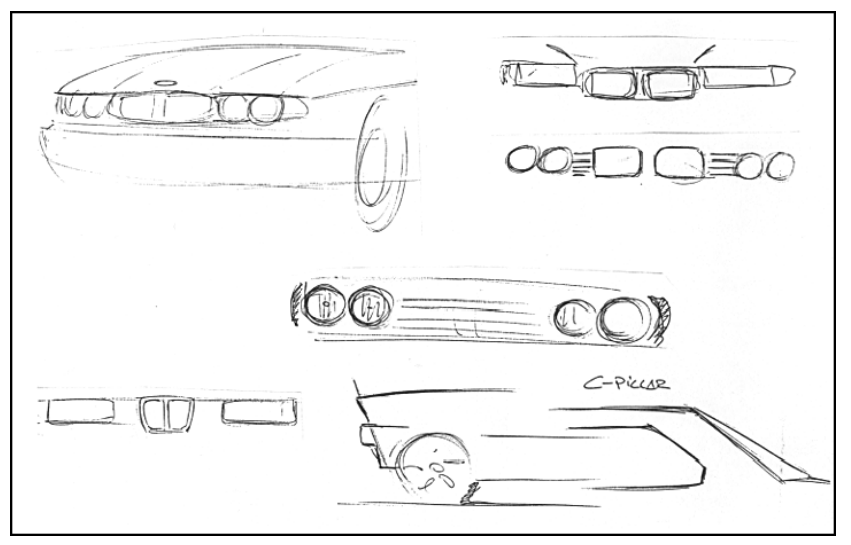




\section{Bouchard, A. Aoussat and R. Duchamp}

We can suppose that all kind of routines could be supported by computer aided styling tools using preexisting databases with the appropriate forms, allowing to concentrate more on the creative part. In fact there is no add value for the designer in drawing a wheel. The originality of a style is more depending on the creative part that will complete the routine tasks. It depends on input data like inspiration sources and on the own routines of the designers. It is true that, to a greater or lesser extent, designers project their own expression through the object. This phenomenon gives partially the originality of a concept, with references that are more or less linked with socially recognised styles. Certain concepts are thus endowed with a signature by which the producer of the shape can be recognised.

The information process in the traditional design activity is a crucial part of the design process. As presented in Section 4.2, designers use a large variety of types of sources coming from different areas as comparable designs, other types of design, images of art, alive beings, objects and phenomena from nature and everyday life. Some researchers emphasised the importance of the sources of inspiration in the building of mental representations (Eckert and Stacey, 2000; Büscher et al., 2002). As a matter of fact, sources of inspiration, references, and influences are an essential base in design thinking, as definition of context, triggers for idea generation, and anchors for structuring designers mental representations of designs (Eckert and Stacey, 2000). They play an important role in the design process, both in defining the context for new designs and in informing the creation of individual designs. Previous designs and other sources of ideas furnish a vocabulary both for thinking about next designs and for describing designs to others (Eckert and Stacey, 2000). The novelty of the design candidates depends mainly of this part, and of the manner to integrate this information during the generation phase too. A specific study showed that creative thinkers usually use more environmental data (data not directly linked to the problem to solve) (Ansburg and Hill, 2002; Bonnardel, 2000).

In fact, the results of the questionnaires showed that the sources of inspiration used for the conceptualisation come from different fields, more or less directly linked with the initial domain of automotive design. They confirm that the inspiration sources play an important role in the design process. Designers could be helped in this phase of information gathering, because they have to manage and categorise a huge number of data. In that sense, some researchers worked on a new image retrieval system which uses specific object names in addition to impression words which can reflect ambiguous human Kansei as retrieval keywords (Kuroda and Hagiwara, 2002). This point of view is best illustrated by the study from Büsher et al. (2002). They suggested the use of a digital space to provide a digital environment in which multimedia inspirational material can be placed.

According to Armi (1991), automotive designers, in order to have control of the complex sculptural forms they are working on, imagine shapes in a mental space while they conceive them. This simulation by visualisation of the imagined real space establishes the field in which the conceptualisation takes place. It can be said that the mental process goes back and forth in this space, as if the designer was trying to grasp it mentally as well as physically. It also appears that the space itself seems to undergo a distortion, a blur, as if ideas oscillated through itself. The visual thinker (Kim et al., 1991) (...) makes several drawings, which are quickly drawn.

Searching and forming an idea does not correspond to a static image, for ideas, which are rarely grasped in a faithful representation: they change and disappear. According to 


\section{Role of sketching in conceptual design of car styling}

Birtley (1995), during the realisation of sketches the space problem corresponds to a goal, which the designer is going to estimate in an almost subconscious way. Hence, it is only by the means of the interpretation of physical visual messages that the designer knows that the image corresponds to the one he had originally intended to make. Schön and Wiggins (1992) emphases that there can be emergent properties in the design medium: each new gesture creates new problems to describe and to solve. All these considerations create a common ground among designers, of which the holistic activity is connected to a wide action of information's searching, hand in hand with artistic competence (Tovey, 1992, 1997). During the making of the final sketches, mental representation consists of a succession of accurate and detailed images (shape, constituents, aspect, impressions linked to the environment, as well as a coloured background or a detailed environment of the vehicle), which offer more acuteness and realism. In spite of a precise definition of the concept by means of sketches, the interpretation of 3D sketches can only be mental, without measurement, and subsequently entails inevitable exaggerations during the realisation. During the 3D modelling, it is only when all the surfaces of the model are clearly defined that the designer exactly imagines the details and constituents that had only been represented until then by simple lines of outline (for example bumper lines). 3D mental images play an important part in the decision process and advising of the designer to the modeller. The natural capacity of the modellers to think in $3 \mathrm{D}$ and to express their own interpretation is an undeniable help. Satisfaction is never absolute or unanimous in regards to the adequacy between the expected image and the one produced, hence, the importance of having deadlines.

The results of the interviews showed that the premature mental image of vehicles or shapes by the designers is very vague and subject to fluctuation. The shape and details change just as a myriad of diverse thoughts would. What is fixed on paper might be a mixture of these different thoughts. The vehicle is thought of as if it were in movement, and is denoted by a dynamic tension. At the earliest stage of the process, neither precise geographic environment nor any details are imagined. Only the global shape that leads to a structure and basic lines are envisioned at this point. The sequence of mental representations is expressed itself partially through the succession of drawn sights from $3 / 4$ left to perspective to $1 / 4$ front to $3 / 4$ right to $1 / 4$ rear, and orthogonal sights. Consistent with this chronology, one might think that the designer imagines a concept that revolves on itself in a move punctuated by mental and graphic explicit fixations. Drawing constitutes the image of the thought (Schön and Wiggins, 1992). This revolution is dictated by a compromise between the information need, which is necessary to completely imagine the volume, and the convenience to construct graphic representations. During this process, appreciation in new figure gives people access to otherwise inaccessible parts of the appreciative system (Schön and Wiggins, 1992).

The operative representation of the problem/solution space refers to a set of normative references, which stems from the designers active cultural collection and are projected in the object (signified references and signifiers: archetypes, proportions, inspiration sources, and spatio-temporal position). From the outset of the project, the designer assimilates the brief's data. During the brainstorming phase, his mental image integrates forms associated to a vehicle type, and usually to a brand. Each designer then orally describes his own representation to the others, which ultimately results in the verbal description of a common representation. The language used often refers to objects or to human beings by analogy. Thus, individual mental interpretation starts to appear on paper. The designers become so impregnated with strong verbal and visual codes 


\section{Bouchard, A. Aoussat and R. Duchamp}

(type, brand image, target consumers ...) that they can materialise with notes or sketches. Here, the sketch constitutes a materialisation that would tend to match what Lebahar (1993) defines as a plan: the means to simulate problems and solutions, and privileged system of spatial data processing. The latter in turn will be completed by an action of information searching, which will enable them to imagine the concept formally. During the making of the first sketches, mental representation is characterised by a succession of global and vague images of the concept (outline, lines, proportions) that go hand in hand with a particular atmosphere (for example, hostile city). Details such as the bumper are not taken into account at this stage of the process in order not to deviate markedly from the global shape or good shape.

The development of the shape and of the spatial configuration during conceptual design follows a dialectic process with the translation of generic rules into specific appearances (Purcell and Gero, 1998). Visual designers have the use of a holistic thinking, and the development of an overall solution proposal conceived as a hole seems to be fundamental in the process (Tovey, 1992, 1997). This first overall point of view is a condition for creativity. The creation of the first sketches corresponds to a first goal, which is the realisation of the good shape, which will be refined later by the addition of details. During this stage, numerous stylistic characteristics are clarified such as dimension, position and orientation. Orientation is a property of the outline of asymmetric forms (Group, 1992), which takes place in our case in a 3D space. Orientation for instance is related to the notion of movement in the sense that it is intimately linked to the notion of balance, which stems from our perception of gravity. By considering this example, shape appears to be an expression correlated to contents in different ways. That is the reason why the role of the context is decisive in the reading of a shape, which is connected to a culturalised type. In our experiments, we tried actually to find out a logical way of use of these semiotic properties, in order to analyse the design sketching.

Graphic representation and more particularly freehand sketches are essential for crystallising ideas in the early stages of design. In design the graphic representations can alternately be considered as the realisation of a part of the problem and a set of means adapted to finding solutions to the problem of definition and of dimensioning of an object (Akin, 1978). Graphic practice intervenes in the upstream phase of the process, as soon as data and knowledge can be understood in spatial terms. It creates a physical visual support for the production and structuring of new ideas. According to Schön and Wiggins (1992), designers use their sketches as a basis for reinterpretation of what have been drawn: this maps on to emergence. Gero (2002) has found that designers when sketching revisit their sketches after a while they sometimes make unexpected discoveries and conclude that sketches serve as a physical setting in which design thoughts are constructed on the fly in a situated way. The resulting realisation stems from the immediate fixation of a mental image dictated as a whole in sketch form. According to Lebahar (1993) the first graphic performances have to be quick enough and limited to the information necessary to solve the predicament, by expressing a minimum set of solutions on the object to be conceived. Elaborated forms stem from the graphic encoding of the constraints which are held in the space problem, as well as the choices that come from the anticipating mental image of an overall picture and finally, from the anticipations of the process. These forms should ensure that the research can still be undertaken without interrupting the design process. In order to do so, the designer keeps the volumes he creates vague as long as possible, the strategy being to specify at the right 


\section{Role of sketching in conceptual design of car styling}

moment. The representation is precise enough so as to express a problem and indistinct enough to enable deformations that will not altogether question its very existence. This explains the elastic and distortable signifiers of its composition (Lebahar, 1993). In the same way, Norman (1991) points out that the form of representation used by an artifact carries great weight in determining its functionality and utility. According to Suwa and Tversky (1996), sketches make apparent to designers not only perceptual features but also inherently non-visual functional relations, allowing them to extract function from perception in sketches. For example the choice of the first particular representation of 3/4 left during the earliest phase of sketching is not arbitrary: it emphases some mappings at the expense of others, makes some explicit and visible, whereas other are neglected, and the physical form suggests and reminds the designer of the set of possible operations. Freehand drawing is an effective method of simulation where each graphic simulation is an operative reversible image. This simulation uses incomplete figures as graphic bases of simulation, which are indistinct and possibly distorted. It also uses a system of signifiers (indications, symbols, icons, and plans) that are appropriate for the combined evocation of concrete objects (parts of vehicles), as well as of abstracted objects (classes of problems). Thus, the research and gradual definition of the object will be made by the realisation of graphic representations, which will be increased in accuracy and in information as a result of the assimilation-accommodation cycle. The quest for certainty is carried out by fixating certitudes, variables, coordinating point of views, and dimensional coordination by comparison.

The manual realisation of sketches is a means to progressively reduce uncertainty. It enables to explore eventual solutions regarding issues such as aesthetic, ergonomic, spatial, technological, and economic difficulties. In this sense, the manual drawing makes it possible to write and erase at the pace of the thought (Sperandio, 1987), thus leaving traces of the mental process of the reasoning. The latter is plastic and appears to be indispensable in the phases of volume search. This gives birth to an analogical relation of the drawings with the concept, thus enabling to manipulate them as transformable mock-ups (Lebahar, 1993). New software should fit with these properties.

\section{Conclusion}

Designers begin usually their work with sketches in order to come up with ideas and concepts, and later turn to using drafting and CAD modelling tools, and to building mockups in order to visualise, compare, implement and validate them. Most available computational tools facilitate the latter process, while few tools aid designers in the former. Given its nature and particular position in the genesis and in the progressive object's construction, nowadays more and more research is undertaken on sketching activities in the hope of integrating this capacity into computer aided styling tools (Scrievener and Clark, 1994).

Our purpose was here is to study the cognitive activity of the designers especially during the conceptual design phase, in order to better appreciate the role of traditional external representations during this phase and to give orientations aimed to develop new digital styling tools dedicated to the conceptual design phase. The first results related to the questionnaires showed the importance of the inspiration sources in the design process, acting as a categories of mental images from general fields, which facilitate the evocation process. Two other categories were identified, more linked with the representation 


\section{Bouchard, A. Aoussat and R. Duchamp}

of the new object, which will emerge, including the use of simple geometric forms. The emergence of new forms and ideas seems to be partially inspired by analogies, with attributes which aren't initially related to the object to design in the current field. Designers could be helped in this phase of information gathering with new image retrieving systems with keywords and images.

According to the results of the protocol study, we can distinguish two main phases during the conceptualisation in car design: a first phase which includes routine activities, depending on a certain level of constraints, and a second phase, more linked with an ill defined part of the object to create, giving a big variety of potential solutions.

The first phase corresponds to the execution of a base expressing the global proportions of the vehicle, by drawing simple forms which are geometrically and mnemonically easy to memorise: circles, ellipses and lines. Even if the strategy can be $2 \mathrm{D}$ or $3 \mathrm{D}$ oriented, the activity includes in both cases a certain level of routine at this stage, and the creative part is relatively limited. In fact, the conceptualisation takes place in a constrained environment, which impose certain parts of the vehicle and its representation.

The second phase corresponds to the elaboration of the silhouette, which gives the character of the vehicle, very differentiated. This phase is more creative and linked with the notion of emergence emphasised by Schön and Gero.

We can suppose that all kind of routines could be helped by computer aided styling tools using preexisting databases with the appropriate forms, allowing to concentrate more on the creative part.

As a perspective, it may be relevant to extend this kind of experiments to other fields of industrial design, in order to try to define modules for Computer Aided Design Tools, with preexisting objects in databases helping in the most constrained and routine parts.

\section{Acknowledgements}

This work was supported by a EUNET grant of the National Technical Research Association. The author would like to acknowledge support from the Coventry University (John Owen, Neil Birtley and Samantha Porter) and of Marilyn Roberty (independent psychologist) and the participants to this research.

\section{References}

Akin, Ö. (1978) 'How do architects design?', in Latombe, J.C. (Ed.): Artificial Intelligence and Pattern Recognition in Computer-Aided Design, North Holland, New York, pp.65-104.

Ansburg, P.I. and Hill, K. (2002) 'Creative and analytic thinkers differ in their use of attentional resources', PAID, Vol. 34, No. 7, May 2003, pp.1141-1152.

Armi, C.E. (1991) The Art of American Car Design: The Profession and Personalities: 'Not Simple Like Simon', Pennsylvania State University Press, ISBN: 0271004797.

Birtley, N. (1995) Internal Research Report, Coventry University, Priory Street, Coventry CV1 5FB.

Bisseret, A., Figeac-Letang, C. and Falzon, P. (1988) 'Modelling opportunistic reasonnings: the cognitive activity of traffic signal setting technicians', Rapport de Recherche INRIA No. 893, Rocquencourt. 


\section{Role of sketching in conceptual design of car styling}

Bonnardel, N. (2000) 'Towards understanding and supporting creativity in design: analogies in a constrained cognitive environment', KBS, Vol. 13, pp.505-513.

Bouchard, C. and Aoussat, A. (2002) 'Modelization of the car design process', International Journal of Vehicle Design, Vol. 31, No. 1, pp.1-10.

Bouchard, C. (1997) Modelling the Car Design Process. A Design Watch Method Applied to the Design of Visual Components, Thesis, pp.1-10.

Büscher, M., Frielaender, V., Hodgson, E., Rank, S. and Shapiro, D. (2002) 'Design on objects: imaginative practice, aesthetic categorization, and the design of multimedia archiving support', Digital Creativity, Vol. 11, No. 3, pp.161-172.

Carrol, J.M. and Moran, T.P. (1991) 'Introduction to a special issue on design rationale', Human-Computer Interaction, Vol. 6, pp.197-200.

Eckert, C. and Stacey, M. (2000) 'Sources of inspiration: a language of design', Design Studies, Vol. 21, No. 5, September, pp.523-538.

Falzon, P. (1969) 'Les activités de conception: réflexions introductives', Performances Humaines et Techniques, dossier 'L'activité des Concepteurs', No. 74, pp.7-11.

Gero, J.S. (2002) 'Towards a theory of designing as situated acts', International Conference 'The Science of Design', Lyon, France, March 15-16.

Group, M.U. (1992) Traité du Signe Visuel, Groupe Mu Ed., Seuil.

Kim, M.C., Chanson, S.T. and Vuong, S.T. (1991) Protocol Trace Analysis Based on Formal Specifications, FORTE, pp.393-408.

Kuroda, K. and Hagiwara, M. (2002) 'An image retrieval system by impression words and specific objects names - IRIS', Neurocomputing, Vol. 43, pp.259-276.

Lebahar, J.C. (1992) 'Quelques formes de Planifications Significatives de l'activité de conception en design industriel', Le Travail Humain, Tome 55, No. 4, pp.329-351.

Lebahar, J.C. (1993) 'Aspects cognitifs du travail du designer industriel', Design Recherche, No. 3, Février, pp.9-20.

Norman, D.A. (1991) 'Cognitive artifacts in designing interaction: psychology at the human-computer interface', J.M. Caroll., Cambridge University Press, Cambridge UK, pp.17-38.

Ochanine, D.A. (2001) 'The operative image of controlled object in 'Man-Automatic Machine' systems', in Leplat, J. (Dir.): L'analyse du Travail en Psychologie Ergonomique (Tome I), Octarès Éditions, $2^{\mathrm{e}}$ éd., Toulouse.

Piaget, J. (1948) Die Entwicklung des Räumlichen Denkens Beim Kinde, Ernst Klett Verlag, ISBN 31291601 .

Purcell, A.T. and Gero, J.S. (1998) 'Drawings and the design process', Design Studies, Vol. 19, pp.389-430.

Richard, J.F. (1983) Functioning Logic and using Logic, INRIA Report No. 202, April.

Römer, A., Weisshahn, G. and Hacker, W. (2001) 'Effort-saving product representations in design results of a questionnaire survey', Design Studies, Vol. 22, January, pp.473-491.

Schön, D.A. and Wiggins, G. (1992) 'Kinds of seeing and their functions in designing', Design Studies, Vol. 13, No. 2, pp.135-156.

Scrievener, S.A.R. and Clark, S. (1994) 'Sketching in collaborative design', in MacDonald, L. and Vince, J. (Eds.): Interacting with Virtual Environments, Wiley Professional Computing, England.

Sperandio, J.C. (1987) La Psychologie en Ergonomie, Paris PUF.

Suwa, M. and Tversky, B. (1996) 'What architects see in their sketches: implications for design tools', CHI Electronic Proceedings, pp.181-192. 


\section{Bouchard, A. Aoussat and R. Duchamp}

Tovey, M. (1992) 'Intuitive and objective processes in automotive design', Design Studies, Vol. 15, No. 1, January, pp.23-41.

Tovey, M. (1997) 'Styling and design: intuition and analysis in industrial design', Design Studies, Vol. 18, No. 1, January, pp.5-25.

Wang, H. (1995) 'An approach to computer-aided styling', Design Studies, Vol. 16, No. 1, January, pp.50-61. 